\title{
Orbiter Gap Filler Bending Model for Re-entry
}

\author{
Charles H. Campbell*, \\ NASA Johnson Space Center, Houston, TX 77058
}

\begin{abstract}
Pressure loads on a protruding gap filler during an Orbiter reentry are investigated to evaluate the likelihood of extraction due to pressure loads, and to ascertain how much bending will be induced by re-entry pressure loads. Oblique shock wave theory is utilized to develop a representation of the pressure loads induced on a gap filler for the ISSHVFW trajectory, representative of a heavy weight ISS return. A free body diagram is utilized to react the forces induced by the pressure forces. Preliminary results developed using these methods demonstrate that pressure loads, alone, are not likely causes of gap filler extraction during reentry. Assessment of the amount a gap filler will bend over is presented. Implications of gap filler bending during reentry include possible mitigation of early boundary layer transition concerns, uncertainty in ground based measurement of protruding gap fillers from historical Orbiter flight history, and uncertainty in the use of Orbiter gap fillers for boundary layer prediction calibration. Authors will be added to the author list as appropriate.
\end{abstract}

\section{Introduction}

$\mathrm{F}$ ight history on the Orbiter vehicle illustrates that gap fillers can and do come out during the ascent/reentry cycle. Two occurrences of protruding gap fillers, on STS-28 and STS-73, during Orbiter reentry are believed to have caused very early boundary layer transition (BLT), near a mach number of 18. During STS-114, two gap fillers were removed by an Extra Vehicular Activity (EVA) crewmember because of the uncertainty involved in determining if those protuberances at the locations identified on Discovery would lead to early BLT and potential catastrophic consequences during reentry. After Discovery returned from the STS-114 mission, the Orbiter Thermal Protection System (TPS) community engaged in a concerted activity to evaluate the installation processes and design involved with the TPS gap fillers. As a direct result of that STS-114 post-flight activity, significant changes have been made in the gap filler installation process. In addition, due to previous occurrences of protruding gap fillers, and the concern with potential consequences of early BLT as well as the mission impacts to perform additional EVA activities to remove gap fillers, a long term activity to remove and replace gap fillers on the Orbiter fleet has begun. However, due to the large number of gap fillers on the fleet and the time involved with reinstallation of the gap fillers, the TPS vehicle processing is a significant schedule concern. Prioritization of the Orbiter regions to have the gap fillers reinstalled has occurred with significant inputs from the Orbiter reentry technical community. Recent activity by the Space Shuttle Program (SSP) Systems Engineering and Integration (SE\&I) community investigating the risk involved with debris transport of gap fillers has elevated the SSP concern in regard to extraction of gap fillers during ascent. Because of lessons learned during the Return To Flight process in regard to foam and ice loss on ascent, the SSP Shuttle Engineering Review Board (SERB) has requested that engineering analyses be performed on the extraction processes involved with gap filler removal during both Orbiter ascent and reentry. In order to support a simple engineering analyses framework, analytical flow field methods are utilized to develop a representative reentry pressure loading history on a protruding gap filler. A heavy weight trajectory representative of an Internation Space Station (ISS) return mission is utilized to evaluate pressure loads on a protruding gap filler. Loads on the gap filler are assessed in order to evaluate the likelihood that the gap filler can be extracted due to pressure, and the amount that a gap filler may bend due to pressure.

\footnotetext{
* Aerospace Engineer, Aeroscience and Applied CFD Branch, M/C EG3, Senior Member.
} 


\section{Analyses}

An analytical framework for evaluating pressure loads has been assembled in Excel. To the authors knowledge, this model represents the first attempt to model the effects of re-entry pressure loads on an Orbiter protruding gap filler during re-entry. Representative deflection angle of a protruding gap filler is provided to demonstrate that the pressure load model has been implemented. Representative geometry of a gap filler bending due to reentry pressure loads are also included to demonstrate that a simple structural analyses has been implemented to evaluate the geometry of a deflected, protruding gap filler.

The intent of this paper will be to provide a summary of the methodology utilized for determination of the pressure load distribution, assessment of the force balance induced on the gap filler, and an assessment of the geometry of the gap filler during re-entry conditions. This information will be used to understand the possible uncertainty in the height of gap fillers that were documented on the ground for the STS-28 and STS-73 Shuttle missions, which have also been attributed with early boundary layer transition.

\section{Gap Filler Deflection}

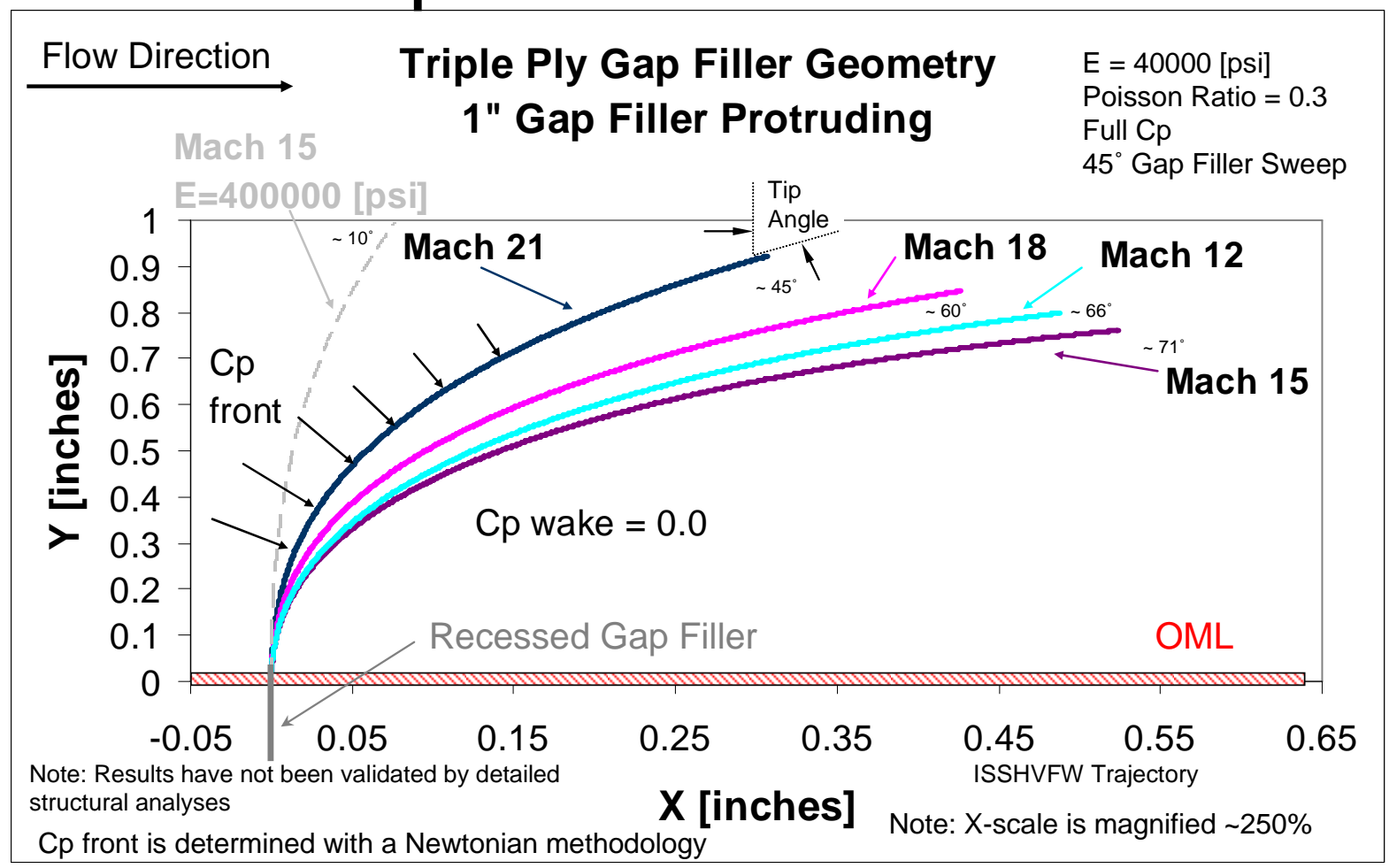




\section{Results}

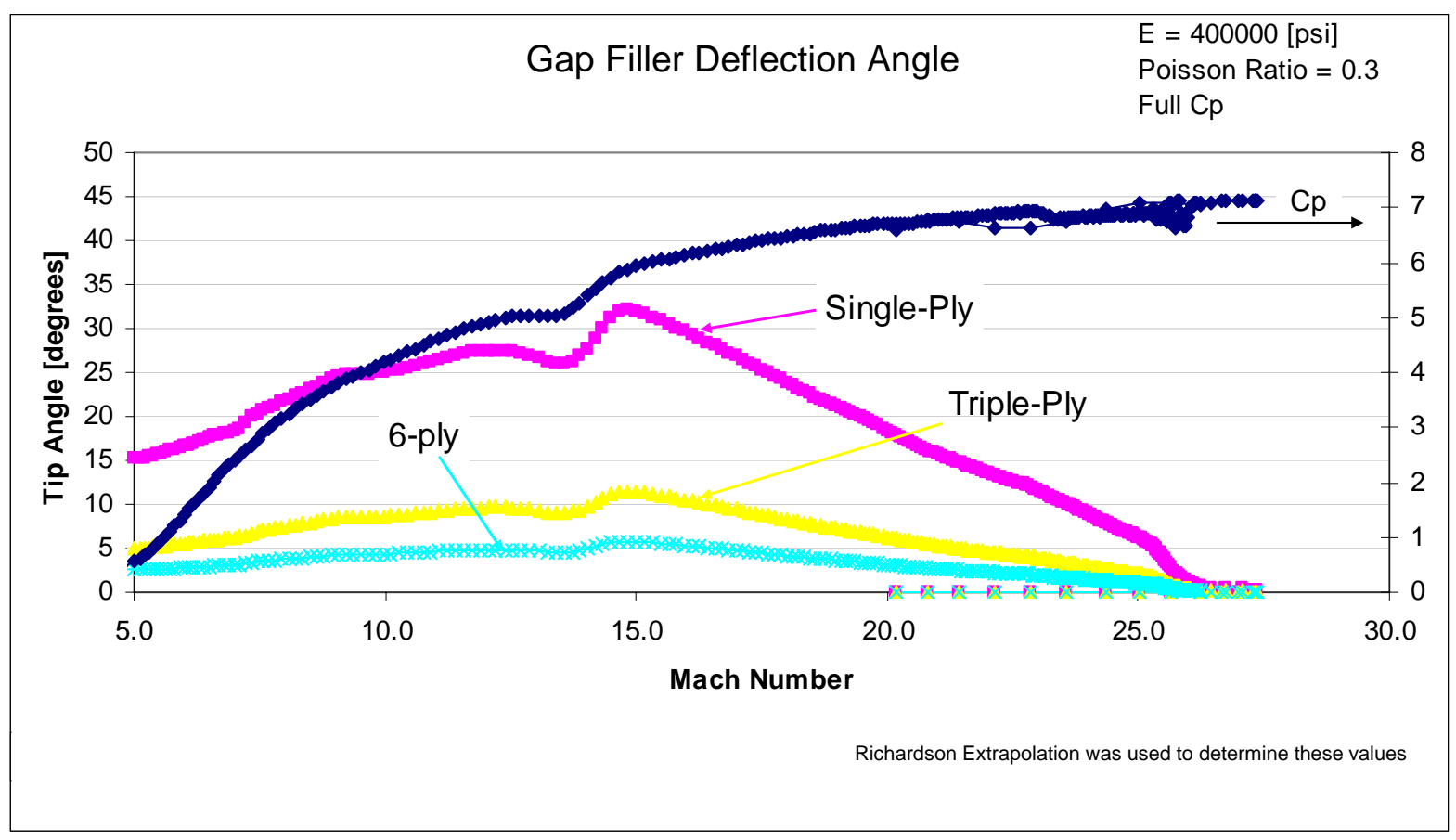

